Acta Crystallographica Section D

\section{Biological Crystallography}

ISSN 1399-0047

Qun Wan, ${ }^{\mathrm{a}, \mathrm{b}}$ Qiu Zhang, ${ }^{\mathrm{a}}$ Scott Hamilton-Brehm, ${ }^{\mathrm{C}}$ Kevin Weiss, ${ }^{\mathrm{a}}$ Marat Mustyakimov, ${ }^{a}$ Leighton Coates, ${ }^{\mathrm{a}}$ Paul Langan, ${ }^{\mathrm{a}}$ David Graham ${ }^{c}$ and Andrey

Kovalevsky ${ }^{\mathrm{a} *}$

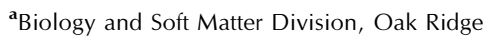
National Laboratory, PO Box 2008, Oak Ridge, TN 37831, USA, 'b Department of Biochemistry, College of Medicine, Yangzhou University, Yangzhou, 225001, People's Republic of China, and ${ }^{\mathbf{c}}$ Biosciences Division, Oak Ridge National Laboratory, PO Box 2008, Oak Ridge, TN 37831, USA

Correspondence e-mail: kovalevskyay@ornl.gov

\title{
X-ray crystallographic studies of family 11 xylanase Michaelis and product complexes: implications for the catalytic mechanism
}

Xylanases catalyze the hydrolysis of plant hemicellulose xylan into oligosaccharides by cleaving the main-chain glycosidic linkages connecting xylose subunits. To study ligand binding and to understand how the $\mathrm{pH}$ constrains the activity of the enzyme, variants of the Trichoderma reesei xylanase were designed to either abolish its activity (E177Q) or to change its $\mathrm{pH}$ optimum $(\mathrm{N} 44 \mathrm{H})$. An E177Q-xylohexaose complex structure was obtained at $1.15 \AA$ resolution which represents a pseudo-Michaelis complex and confirmed the conformational movement of the thumb region owing to ligand binding. Co-crystallization of $\mathrm{N} 44 \mathrm{H}$ with xylohexaose resulted in a hydrolyzed xylotriose bound in the active site. Co-crystallization of the wild-type enzyme with xylopentaose trapped an aglycone xylotriose and a transglycosylated glycone product. Replacing amino acids near Glu177 decreased the xylanase activity but increased the relative activity at alkaline $\mathrm{pH}$. The substrate distortion in the E177Q-xylohexaose structure expands the possible conformational itinerary of this xylose ring during the enzyme-catalyzed xylan-hydrolysis reaction.

\section{Introduction}

The hydrolysis of hemicellulose into its constituent sugars is an important step in the conversion of renewable plant biomass into biofuels and other bioproducts. Hemicelluloses include a diverse series of substituted glucan, mannan and xylan polysaccharides, with the latter being particularly abundant in grasses. The main chain of xylan consists of $\beta$-(1 $\rightarrow 4)$-D-xylose moieties decorated with arabinose, glucuronate, acetyl groups etc. (Scheller \& Ulvskov, 2010). The full degradation of xylan requires the action of several secreted enzymes, called hemicellulases, which include endoxylanases that cleave the $\beta$-1,4-xylosidic linkages and other enzymes that hydrolyze the side-chain substituents (Dodd \& Cann, 2009). The benefits of supplying enzymatic cocktails that include hemicellulases during the hydrolysis of pretreated biomass have been thoroughly established (Gupta et al., 2008; Garlock et al., 2009). However, the current understanding of the xylanase catalytic mechanism and specificity does not fully describe the activity of the enzyme towards long-chain complex substrates; therefore, further molecular research is necessary to optimize the deconstruction of xylan under process-relevant conditions.

The endo-1,4- $\beta$-xylanases (EC 3.2.1.8) have evolved in several glycoside hydrolase families, including the best-studied family 10 and 11 enzymes from fungi and bacteria (Collins et al., 2005). Family 11 enzymes fold into a jelly-roll shape likened to a partially closed right hand (Fig. 1). Several antiparallel $\beta$-strands bend by almost $90^{\circ}$ to produce a substratebinding groove characteristic of family 11 xylanase active sites
Received 26 June 2013 Accepted 22 August 2013

PDB References: E177Q-X6, 4hk8; N44H-X3, 4hk9; ligand-free $\mathrm{N} 44 \mathrm{H}, 4 \mathrm{hkl}$; Xynll-TrisX2-X3, 4hkw; ligand-free $\mathrm{E} 177 \mathrm{Q}$, 4hko 
(Törrönen et al., 1994). Catalysis proceeds with retention of stereochemistry at the anomeric $\mathrm{C}$ atom $\mathrm{C} 1$ of the nonreducing (glycone) portion of the product. Two catalytic Glu residues face each other from opposite sides of the groove at about $6 \AA$ apart (McCarter \& Withers, 1994; Törrönen et al., 1994; Wakarchuk et al., 1994; Fig. 1). The hydrolysis reaction is believed to follow a double-displacement mechanism, with one Glu residue acting as a general acid/base catalyst and the other as a nucleophile (Miao et al., 1994; Davoodi et al., 1995; McIntosh et al., 1996; White \& Rose, 1997). The $\mathrm{p} K_{\mathrm{a}}$ value of

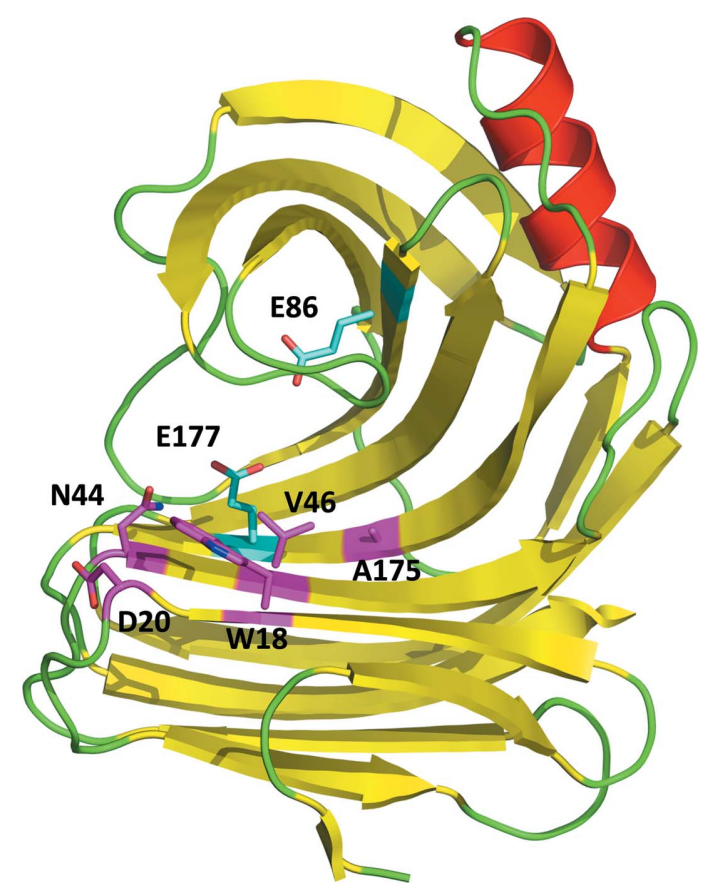

Figure 1

The jelly-roll fold of family 11 xylanase proteins is shown in a cartoon representation colored by secondary structure. The catalytic residues (Glu86 and Glu177 in T. reesei XynII) and the sites of the amino-acid substitutions reported here are shown in stick representation. the acid/base catalyst is necessarily high $(\sim 7)$, while that of the nucleophile is low $(<5)$, in the ligand-free enzyme state and upon formation of the Michaelis complex (Miao et al., 1994; Davoodi et al., 1995). Thus, the former Glu must be protonated and the latter negatively charged to initiate the hydrolysis reaction.

By analogy with hen egg-white lysozyme (Strynadka \& James, 1996) and other retaining glycoside hydrolases (Zechel \& Withers, 2000; Vasella et al., 2002), the protonation of the xylosidic oxygen by the general acid and the attack on $\mathrm{C} 1$ by the nucleophile are proposed to be concerted, producing a transition state with a pentacoordinated $\mathrm{C} 1$ and a strong oxocarbenium character of the $\mathrm{C} 1-\mathrm{O} 5$ bond of the xylose ring. Following decomposition of the transition state, a covalent intermediate forms with the inverted $\mathrm{C} 1$ center directly binding to $\mathrm{C} 1$ of the glycone end of the xylan chain and the carboxylate group of the nucleophile (Davies \& Henrissat, 1995; White \& Rose, 1997; Wakarchuk et al., 1994; Törrönen \& Rouvinen, 1997). In the next stage the reducing (aglycone) portion of the product is thought to leave the active site of the enzyme before the next deglycosylation reaction step. The formation of the intermediate would lead to a drop in the $\mathrm{p} K_{\mathrm{a}}$ of the carboxylic group of the acid/base catalyst by greater than $2 \mathrm{pH}$ units (McIntosh et al., 1996). In the deglycosylation step, this Glu can therefore act as a general base to activate an incoming water molecule, which attacks $\mathrm{C} 1$ of the glycosylenzyme intermediate to break the ester bond to the enzyme, releasing the glycone product. There is X-ray crystallographic evidence for the production of the covalent intermediate in family 10 and 11 enzymes from experiments using activated, chemically modified and unmodified xylooligosaccharides (Havukainen et al., 1996; Notenboom, Birsan, Nitz et al., 1998; Notenboom, Birsan, Warren et al., 1998; Sabini et al., 2001; Sidhu et al., 1999; Suzuki et al., 2009). However, the critical Michaelis complex of family 11 xylanases has not been fully characterized in a high-resolution structure (Vandermarliere et al., 2008).

In order to further investigate the mechanism of these enzymes, we have studied the family 11 xylanase (XynII) secreted by the filamentous fungus Trichoderma reesei (Törrönen et al., 1992). In XynII, Glu177 is the catalytic acid/base residue, whereas Glu86 is the nucleophile (Fig. 1). To abolish the activity of the enzyme, we replaced Glu177 with glutamine in a heterologously expressed protein to form the E177Q variant, effectively eliminating the proton-donating ability of this residue (Wakarchuk et al., 1994; Zolotnitsky et al., 2004). In the $1.15 \AA$ resolution X-ray structure of E177Q co-crystallized with xylohexaose (X6; Fig. 2) at $\mathrm{pH} 7.0$, the intact oligosaccharide is observed within the activesite cavity. We refer to this complex as 
E177Q-X6. Each xylose subunit of X6 is unambiguously observed in the electron-density maps with low atomic displacement parameters ( $B$ factors), allowing the identification of six sugar-binding subsites spanning positions -3 to +3 and making a number of interactions with the xylose subunits (Davies et al., 1997). We also attempted to alter the $\mathrm{pH}$ optimum of the xylanase activity by substituting asparagine at position 44 , which is located near Glu177, with histidine to produce the $\mathrm{N} 44 \mathrm{H}$ variant. In the $1.55 \AA$ resolution X-ray structure of $\mathrm{N} 44 \mathrm{H}$ co-crystallized with $\mathrm{X} 6$ at $\mathrm{pH}$ 6.0, the glycone xylotriose (X3; Fig. 2) portion of the oligosaccharide is present in the active site but not the aglycone portion. We refer to this product complex as $\mathrm{N} 44 \mathrm{H}-\mathrm{X} 3$. The significant reduction in activity of $\mathrm{N} 44 \mathrm{H}$ relative to the wild-type (WT) enzyme may have been key to enabling crystallization of the $\mathrm{N} 44 \mathrm{H}-\mathrm{X} 3$ product complex. In the $1.60 \AA$ resolution X-ray structure of native XynII co-crystallized with xylopentaose (X5) at a higher $\mathrm{pH}$ of 8.5, using tris(hydroxymethyl)aminomethane (Tris) buffer, the transglycosylation reaction product (TrisX2; Fig. 2) and the aglycone product (X3) are both present in the active site. We refer to this structure as XynIITrisX2-X3. In the transglycosylation product a Tris molecule is attached to the anomeric $\mathrm{C} 1$ atom of the glycone xylobiose through a hydroxyl group. Although glycoside hydrolases are used to synthesize alkyl glycosides by transglycosylation (van Rantwijk et al., 1999), no free alkyl glycoside has previously been observed in subsites $-1,-2$ and -3 in a crystal structure. The high-resolution X-ray structures reported here markedly improve our knowledge of substrate and product binding in family 11 xylanases and in addition shed new light on the mechanism of retaining glycosyl hydrolases. The enzyme kinetic measurements using XynII variants demonstrated that the $\mathrm{pH}$ optimum for XynII can be altered by changing steric and hydrophobic interactions near the active-site Glu177 residue, albeit at the cost of decreased xylan-hydrolyzing activity compared with the wild-type enzyme.

\section{Materials and methods}

\subsection{Protein expression and purification}

The details of protein expression and purification for crystallization have been published previously (Wan et al., 2013). Briefly, genes encoding amino-acid positions 2-190 of the native secreted $T$. reesei XynII protein (or its variants) were synthesized by DNA 2.0 (Menlo Park, California, USA) in the pJexpress401 vector with DNA sequences optimized for expression in Escherichia coli. The proteins were expressed in BL21-Gold cells (Agilent Technologies, Santa Clara, California) in Enfors minimal medium (Törnkvist et al., 1996) and were purified using SP cation-exchange chromatography followed by size-exclusion chromatography. The proteins were concentrated to $30-40 \mathrm{mg} \mathrm{ml}^{-1}$ in $0.1 M$ Tris- $\mathrm{HCl}, 0.1 \mathrm{M} \mathrm{NaCl}$ $\mathrm{pH} 8.5$ buffer for crystallization. The native WT protein was purchased from Hampton Research but was dialyzed against $25 \mathrm{mM}$ Tris- $\mathrm{HCl} \mathrm{pH} 8.5$ before crystallization with xylopentaose.
For kinetic analysis, a gene encoding the same amino-acid positions with a hexahistidine ( $\mathrm{His}_{6}$ ) tag attached to the carboxy-terminus was synthesized in the pJexpress401 vector (DNA 2.0, Menlo Park, California, USA). For comparison, purified native XynII from T. longibrachiatum was purchased from Hampton Research (Aliso Viejo, California, USA). This protein shares the same amino-acid sequence as its $T$. reesei ortholog and is considered to be equivalent (Wan et al., 2013).

Deoxyoligonucleotide primers synthesized by Integrated DNA Technologies (Coralville, Iowa, USA) were used to produce site-directed mutations in the synthetic WT gene using the QuikChange II XL kit (Agilent Technologies) according to the manufacturer's instructions (Supplementary Tables $\mathbf{S} 1$ and $\mathbf{S 2}^{\mathbf{1}}$ ). Mutations were confirmed by DNA sequencing at the University of Tennessee Knoxville Molecular Biology Resource Facility. Expression vectors were transformed into E. coli BL21 cells and protein expression, cell lysis, nickel-affinity chromatography, protein concentration and total protein analysis were performed using standard methods (Drevland et al., 2007).

\subsection{Steady-state enzyme rate measurements}

Continuous assays of xylanase activity were performed by measuring the rate of 4-nitrophenyl- $\beta$-D-xylobioside $\left(\mathrm{PNPX}_{2}\right)$ hydrolysis (Megazyme, Wicklow, Ireland; Taguchi et al., 1996). Solutions $(920 \mu \mathrm{l})$ containing $100 \mathrm{mM}$ sodium phosphate buffer were equilibrated in glass cuvettes at $323 \mathrm{~K}$ in a Beckman DU-800 spectrophotometer with a High Performance Temperature Controller. Aliquots of substrate (30 $\mu \mathrm{l}$ $10 \mathrm{mM} \mathrm{PNPX}_{2}$ in methanol) and purified protein $(50 \mu \mathrm{l})$ were added with mixing and the increase in absorbance at $400 \mathrm{~nm}$ was measured for $3 \mathrm{~min}$. The initial slopes of the time-course measurements were used to calculate the initial rate constants. The $\mathrm{pH}$ of the buffer solutions was adjusted using varying proportions of monobasic and dibasic sodium phosphate. Molar absorption coefficients for the 4-nitrophenol product were measured at each $\mathrm{pH}$ value.

Discontinuous assays of xylanase activity were performed using the 3,5-dinitrosalicylic acid (DNS) reagent to determine the concentration of reducing groups following enzymatic hydrolysis (Bailey et al., 1992). The DNS reagent consisted of $1 \%(w / w)$ DNS and $30 \%(w / w)$ sodium potassium tartrate tetrahydrate in $0.4 M$ sodium hydroxide. Three replicates of reaction mixtures $(150 \mu \mathrm{l})$ consisting of $100 \mathrm{~m} M$ sodium phosphate buffer, Beechwood xylan (1-10 $\mathrm{mg} \mathrm{ml}^{-1}$; SigmaAldrich) and purified protein were incubated for $30 \mathrm{~min}$ at $323 \mathrm{~K}$ in microplates. Reactions were terminated by the addition of $150 \mu \mathrm{l}$ DNS reagent and heating for $10 \mathrm{~min}$ at $371 \mathrm{~K}$. The absorbance at $540 \mathrm{~nm}$ of the cooled mixture was determined using a SynergyMx microplate reader (BioTek, Winooski, Vermont, USA). A standard curve (from control reactions containing $0-1 \mathrm{mg} \mathrm{ml}^{-1}$ D-xylose) was used to calculate reducing-sugar concentrations. The rate of enzymecatalyzed hydrolysis was calculated after subtracting the

\footnotetext{
${ }^{1}$ Supporting information has been deposited in the IUCr electronic archive (Reference: BE5239).
} 
Table 1

Steady-state kinetic parameters for WT and variant XynII proteins.

The initial rates measured for xylan hydrolysis were fitted to a sigmoidal model of cooperativity, except as indicated. The substrate concentration at which $v=0.5 \mathrm{~V}\left(K_{1 / 2}\right)$, the turnover number $\left(k_{\mathrm{cat}}\right)$ and the Hill coefficient $(h)$ are listed for each model. WT is the native WT protein and rWT is the recombinant enzyme.

\begin{tabular}{|c|c|c|c|c|c|c|c|c|c|c|}
\hline \multirow[b]{2}{*}{ Enzyme } & \multirow[b]{2}{*}{$\begin{array}{l}\text { Protein } \\
\text { concentration } \\
\left(\mu \mathrm{g} \mathrm{ml}^{-1}\right)\end{array}$} & \multicolumn{3}{|l|}{ pH 5} & \multicolumn{3}{|l|}{ pH 6} & \multicolumn{3}{|l|}{ pH 7} \\
\hline & & $\begin{array}{l}K_{1 / 2} \\
\left(\mathrm{mg} \mathrm{ml}^{-1}\right)\end{array}$ & $\begin{array}{l}k_{\text {cat }} \\
\left(\mathrm{s}^{-1}\right)\end{array}$ & $h$ & $\begin{array}{l}K_{1 / 2} \\
\left(\mathrm{mg} \mathrm{ml}^{-1}\right)\end{array}$ & $\begin{array}{l}k_{\mathrm{cat}} \\
\left(\mathrm{s}^{-1}\right)\end{array}$ & $h$ & $\begin{array}{l}K_{1 / 2} \\
\left(\mathrm{mg} \mathrm{ml}^{-1}\right)\end{array}$ & $\begin{array}{l}k_{\text {cat }} \\
\left(\mathrm{s}^{-1}\right)\end{array}$ & $h$ \\
\hline WT & 0.2 & 2.53 & 82.5 & 1.8 & 3.97 & 126 & 1.3 & 7.48 & 142 & 1.6 \\
\hline rWT & 0.2 & 3.17 & 90.4 & 1.5 & 5.36 & 147 & 1.5 & 8.10 & 150 & 2.3 \\
\hline N44V & 1.0 & $14.3 \dagger$ & $52.7 \dagger$ & & $10.7 \dagger$ & $48.1 \dagger$ & & 10.2 & 48.6 & 1.3 \\
\hline N44V & 0.5 & $11.7 \dagger$ & $41.6 \dagger$ & & $14.5 \dagger$ & $65.5 \dagger$ & & 18.9 & 108 & 1.4 \\
\hline N44D & 1.0 & 4.32 & 12.6 & 1.9 & 4.48 & 14.1 & 2.1 & 6.67 & 8.95 & 3.5 \\
\hline A175S & 1.0 & 4.11 & 59.7 & 1.6 & 10.9 & 107 & 1.3 & & & \\
\hline A175S & 0.2 & 6.48 & 58.3 & 1.6 & 5.40 & 58.0 & 1.5 & 7.55 & 66.3 & 1.7 \\
\hline V46L & 2.0 & & & & $39.42 \dagger$ & $113 \dagger$ & & 37.2 & 141 & 1.1 \\
\hline
\end{tabular}

$\dagger$ Data from these conditions were better fitted using the Michaelis-Menten-Henri equation. The parameters listed are the $K_{\mathrm{m}}$ and $k_{\text {cat }}$ values. $\quad$ \$ The kinetic parameters for the V46L enzyme at pH 8 were $K_{1 / 2}=5.59 \mathrm{mg} \mathrm{ml}{ }^{-1}, k_{\text {cat }}=20.8 \mathrm{~s}^{-1}$ and $h=2.0$.

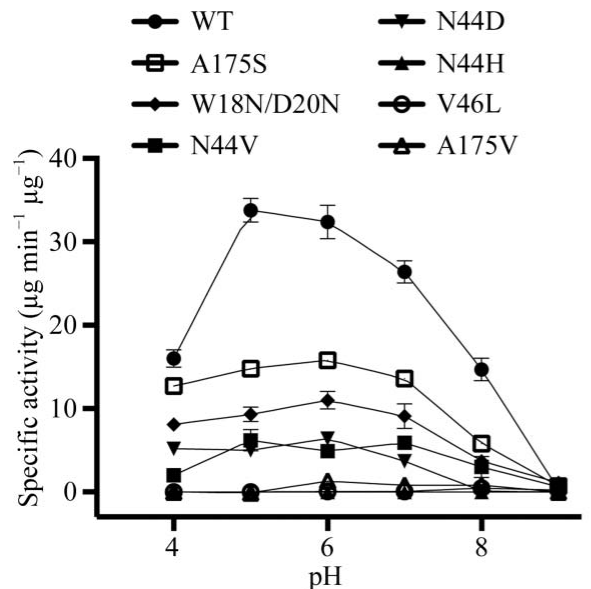

(a)

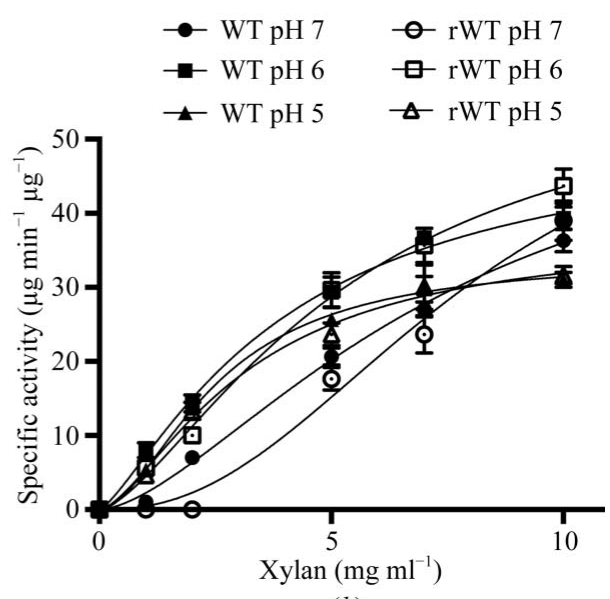

(b)
Figure 3

Steady-state rates of beechwood xylan hydrolysis. (a) The XynII variants demonstrated lower specific activities in all $\mathrm{pH}$ conditions, although the relative rates were more uniform from $\mathrm{pH} 4$ to $\mathrm{pH}$ 7. The reaction mixtures contained $5 \mathrm{mg} \mathrm{ml}^{-1}$ xylan. (b) The substrate-saturation curves for the WT and rWT enzymes demonstrate their kinetic equivalence. The rate data are best fitted using a sigmoidal kinetics model.

background concentrations of reducing sugars measured in control reactions incubated without enzyme. Low concentrations, $0.2 \mu \mathrm{g} \mathrm{ml}^{-1}$ wild-type or $1.0 \mu \mathrm{g} \mathrm{ml}^{-1}$ variant xylanase, were selected from the linear portion of a plot of reducingsugar product versus enzyme concentration.

Initial rate data from continuous assays and discontinuous assays were fitted with Michaelis-Menten-Henri (MMH) or allosteric sigmoidal equations by nonlinear regression (GraphPad Prism v.6.0; GraphPad Software). Data from discontinuous assays of the WT enzyme (Hampton Research) at each $\mathrm{pH}$ condition were better fitted by the allosteric sigmoidal model compared with the MMH model $(\mathrm{pH} 5, P<$ 0.001 ; pH 6, $P=0.0264$; pH 7, $P=0.0002$; Figs. $3 a, 3 b$ and Table 1). Therefore, the allosteric sigmoidal equation was used to fit all kinetic data, except for the rates measured at $\mathrm{pH} 5$ and 6 for the N44V variant, where the estimated Hill coefficient was close to 1.0 and $\mathrm{MMH}$ was the preferred model.

\subsection{Crystallization and data processing}

The WT and all variants were crystallized using the hanging-drop method: $1 \mu \mathrm{l}$ protein solution was added to $1 \mu \mathrm{l}$ reservoir solution and equilibrated against $0.5 \mathrm{ml}$ reservoir solution. For the variants in the ligand-free state, the reservoir solution consisted of 15-20\% PEG 8000, 0.2 $M$ NaI, 0.1 $M$ MES$\mathrm{NaOH} \mathrm{pH}$ 6.0. For the E177Q-X6 complex, the reservoir solution consisted of $0.2 \mathrm{M}$ ammonium citrate tribasic pH 7.0, 20\% PEG 3350. For the N44H-X3 complex, the reservoir solution consisted of $18 \%$ PEG 8000, $0.2 \mathrm{M}$ $\mathrm{CaCl}_{2}, 0.1 M$ MES-NaOH pH 6.0. For the XynII-TrisX2-X3 complex the reservoir solution consisted of $20 \%$ PEG 8000, 0.2 $\mathrm{M} \mathrm{CaCl}_{2}, 0.1 \mathrm{M}$ Tris- $\mathrm{HCl}$ $\mathrm{pH}$ 8.5. Data sets were collected on beamline 19-ID at the Advanced Photon Source (Argonne National Laboratory) and were processed with HKL-2000 (Otwinowski \& Minor, 1997).

\subsection{Structure determination, refinement and analysis}

All of the structures were solved by the molecular-replacement method using the program Phaser (McCoy et al., 2007) as incorporated in the PHENIX program suite (Adams et al., 2010). The initial molecular-replacement model was from PDB entry $2 \mathrm{dfb}$ (Watanabe et al., 2006) with all waters and nonbonded ions removed. After a few rounds of refinement using phenix.refine (Afonine et al., 2012) interspersed with manual model building using Coot (Emsley et al., 2010), electron density for the ligands was clearly visible in both the $2 F_{\mathrm{o}}-F_{\mathrm{c}}$ map and the $F_{\mathrm{o}}-F_{\mathrm{c}}$ map. Xylose units were individually placed into the density and refined against the data. In the ligand-free structures, iodine ions were identified according to the anomalous difference maps. Ramachandran plot analysis was performed using MolProbity (Chen et al., 2010). The statistics of data processing and structure refinement are shown in Supplementary Table S3. Figures were generated using PyMOL (Schrödinger, Rockville, Maryland, USA).

Structure alignment was performed using SSM (Krissinel \& Henrick, 2004) as incorporated in Coot. The solvent-accessible areas of the ligands were calculated using AREAIMOL (Saff \& Kuijlaars, 1997) as incorporated in CCP4 (Winn et al., 2011). Xylose conformation and puckering parameters were calculated according to the Cremer-Pople formalism (Jeffrey \& Yates, 1979) and were compared with the dihedral angles of 
Table 2

Xylose conformation parameters for the E177Q-X6, N44H-X3 and XynII-TrisX2-X3 complexes.

The values shown in bold indicate significant departure of the xylose subunit conformation from the common ${ }^{4} C_{1}$ chair.

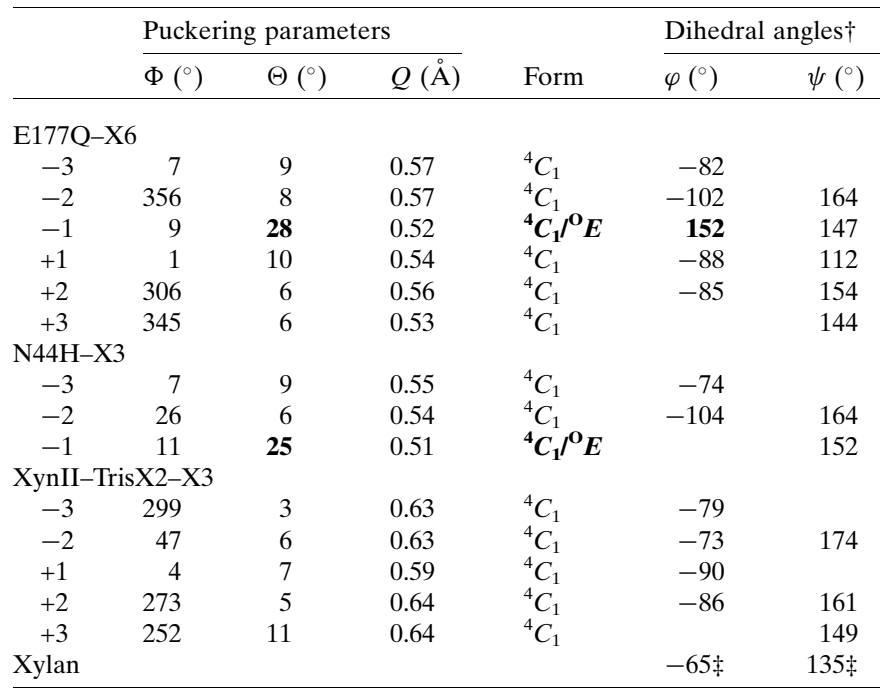

$\dagger$ The dihedral angles are defined as $\varphi\left(\mathrm{O}_{i}-\mathrm{Cl}_{i}-\mathrm{O}_{i+1}-\mathrm{C}_{i+1}\right)$ and $\psi\left(\mathrm{Cl}_{i}-\mathrm{O}_{i+1}-\right.$ $\left.\mathrm{C}_{i+1}-\mathrm{C}_{i+1}\right)$. $\neq$ Values for xylan from De Vos et al. (2006) for comparison.

xylose units in xylan (Table 2; Supplementary Fig. S1). An r.m.s.d. difference plot comparing the structures studied is shown in Supplementary Fig. S2.

Atomic coordinates and structure factors have been deposited in the Protein Data Bank as entries $4 \mathrm{hk} 8$ for E177Q-X6, 4hk9 for $\mathrm{N} 44 \mathrm{H}-\mathrm{X} 3$, 4hkl for ligand-free $\mathrm{N} 44 \mathrm{H}$, 4hkw for XynII-TrisX2-X3 and 4hko for ligand-free E177Q.

\section{Results}

\subsection{Kinetic analysis of xylanase activity using protein variants}

The Glu86 and Glu177 residues are universally conserved in xylanase homologs and cannot be replaced without dramatically impairing enzyme turnover (Ko et al., 1992). Therefore, in order to probe $\mathrm{pH}$-dependent effects on these key catalytic moieties, we replaced the adjacent amino-acid side chains by site-directed mutagenesis and heterologously expressed and purified the protein variants for kinetic analysis. The Asn44 side chain (Fig. 1) forms a hydrogen bond that raises the $\mathrm{p} K_{\mathrm{a}}$ of the Glu177 carboxylate (Törrönen \& Rouvinen, 1995). In acidophilic xylanases, this Asn is replaced by an Asp (Fushinobu et al., 1998) and the same substitution engineered in a neutrophilic bacterial xylanase caused enhanced activity at acidic $\mathrm{pH}$ (Joshi et al., 2000). Two substitutions of this residue, N44V and N44D, in T. reesei XynII significantly decreased the xylan hydrolytic activity (18 and $14 \%$ activity, respectively), although the N44D variant had a higher relative activity at acidic $\mathrm{pH}$, as reported in other systems (Supplementary Fig. S3a; Joshi et al., 2000). An N44H variant had no detectable xylan-hydrolyzing activity under any $\mathrm{pH}$ condition (Fig. $3 a$ ). A recent NMR spectroscopic study demonstrated a lower $\mathrm{pH}$ optimum for the activity of the equivalent $\mathrm{N} 35 \mathrm{H}$ variant of Bacillus circulans xylanase using $\mathrm{PNPX}_{2}$ (Ludwiczek et al., 2013). In our study, residual PNPX $_{2}$ hydrolytic activity was also detected at $\mathrm{pH} 5$ for all three variants (Supplementary Fig. S3b), establishing that glycosidic bond cleavage could occur in the active site. The N44H and N44D mutations probably altered electrostatic interactions with Tyr179 near the +3 site and introduced new nonproductive interactions with the substrate that reduced the turnover. The $\mathrm{pH}$-dependence of hydrolytic activity was notably higher in PNPX $_{2}$ assays, confirming the need to measure xylanase activity using xylan substrates and temperature-insensitive $\mathrm{pH}$ buffers to obtain process-relevant rate constants (Gibbs et al., 2010).

The Val46 side chain (Fig. 1) abuts Glu177 and Glu86 in the active site. To determine the effects of increased van der Waals interactions and hydrophobicity in the active site, we constructed the V46L variant. This protein demonstrated very low levels of xylanase activity under all $\mathrm{pH}$ conditions; however, its relative activity was highest under alkaline conditions $(0.5 \pm 0.2 \mu \mathrm{g}$ xylose per minute per microgram of protein at $\mathrm{pH}$ 8; Supplementary Fig. S3a). Thus, increased hydrophobicity in the variant active site may have increased the $\mathrm{p} K_{\mathrm{a}}$ of Glu177. This Leu substitution occurs naturally in catalytically competent xylanase homologs from Bacillus spp.; therefore, subtle conformational changes may determine its steric interaction with nearby residues. Similarly, the methyl group of Ala175 is $\sim 4 \AA$ from the Val46 isopropyl group. Two variants, $\mathrm{A} 175 \mathrm{~S}$ and $\mathrm{A} 175 \mathrm{~V}$, are predicted to increase the steric interactions with Val46. A175S, which should have fewer steric interactions than $\mathrm{A} 175 \mathrm{~V}$, demonstrated a uniform xylan hydrolytic activity from $\mathrm{pH} 4$ to 7 at about $43 \%$ of that of the WT activity owing to a decreased turnover rate. The A175V variant was significantly impaired, but demonstrated relatively higher activity from $\mathrm{pH} 6$ to $\mathrm{pH} 8$ compared with the WT (Supplementary Fig. S3a). The A175V protein demonstrated very low levels of $\mathrm{PNPX}_{2}$ hydrolytic activity at all $\mathrm{pH}$ values. This residue is often replaced by bulkier side chains such as Thr in acidophilic xylanases, and these results are consistent with an increase in the $\mathrm{p} K_{\mathrm{a}}$ of the Glu177 carboxylate.

The Trp18 and Asp20 residues of XynII are replaced by Asn in the acidophilic xylanases from Scytalidium acidophilum and Aspergillus niger. A W18N/D20N variant of XynII demonstrated nearly uniform xylan hydrolytic activity from $\mathrm{pH} 4$ to $\mathrm{pH} 7$, although its activity at $\mathrm{pH} 5$ was only $28 \%$ of that of the WT. The role of these residues is unclear, but the indole side chain of Trp18 may influence the conformation of the nearby Asn 44 side chain $(3.7 \AA)$ through steric interactions.

The native and heterologously expressed WT xylanases catalyzed the hydrolysis of beechwood xylan suspensions with similar rate constants (Fig. $3 b$ ). In these $30 \mathrm{~min}$ reactions $2-4 \%$ of the xylan substrate was hydrolysed, and corrected measurements of the reducing-sugar products were used to estimate the initial rates. Rather than the expected hyperbolic model of substrate-saturation kinetics, both enzymes demonstrated a sigmoidal relationship between substrate concentration and initial rate, which was most prominent at $\mathrm{pH} 7$. Most variant protein rates were also best fitted by a sigmoidal 
model, except for the $\mathrm{N} 44 \mathrm{~V}$ protein at acidic $\mathrm{pH}$, where a hyperbolic model was sufficient to fit the data (Table 1). At pH values below the optimum the turnover of the WT enzyme

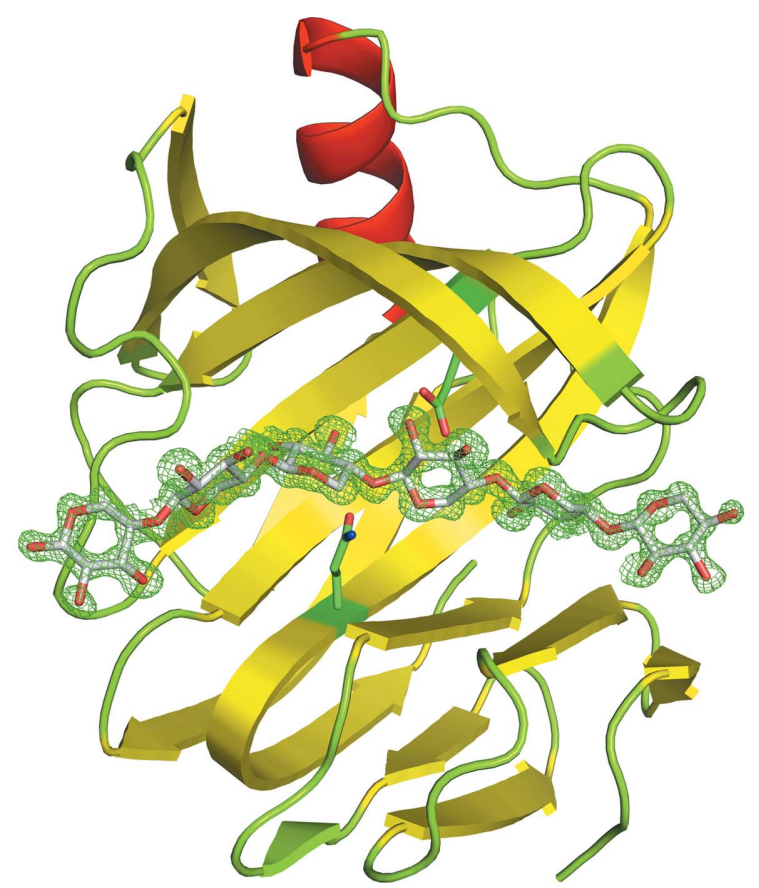

(a)

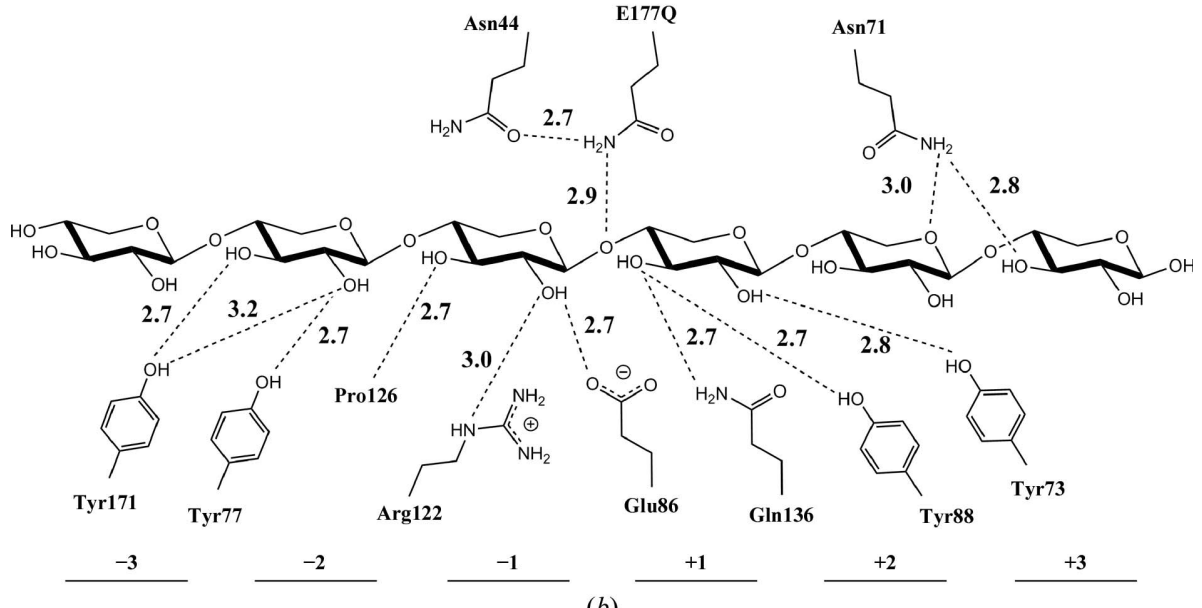

(b)

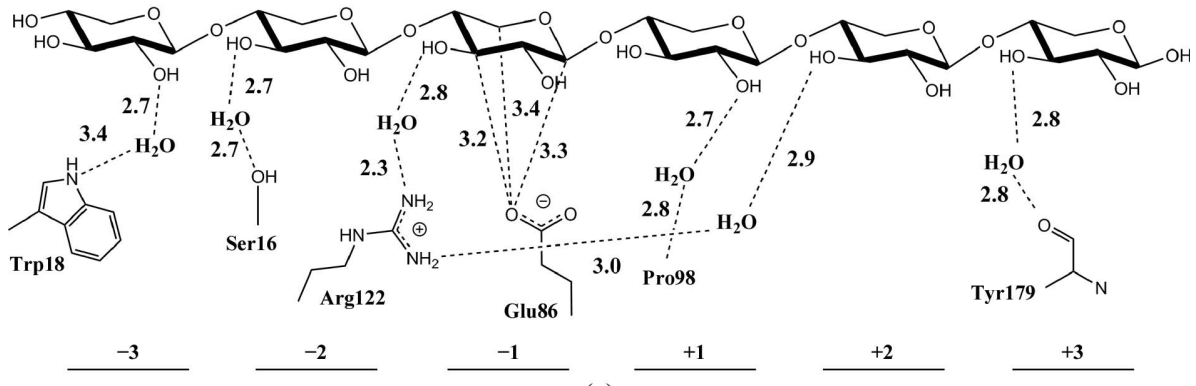

Figure 4

(c)

(a) OMIT difference $2 F_{\mathrm{o}}-F_{\mathrm{c}}$ electron density of xylohexaose in E177Q-X6 contoured at the $1.0 \sigma$ level. (b) Chemical diagram of conventional direct hydrogen-bond interactions between X6 and E177Q residues. (c) Water-mediated interactions between X6 and the active-site residues and the unconventional $\mathrm{C}-\mathrm{H} \cdots \mathrm{O}$ hydrogen bonds between Glu86 and the -1 xylose subunit in E177QX6. decreased, while at $\mathrm{pH}$ values above the optimum $K_{1 / 2}$ increased and the cooperativity (represented by the Hill constant $h$ ) increased. This sigmoidal response was not observed using the soluble $\mathrm{PNPX}_{2}$ substrate in continuous assays; therefore, this apparent cooperative behavior is specific to longer chain substrates.

\subsection{Structure of the E177Q-X6 pseudo-Michaelis complex}

Substitution of the Glu177 carboxyl group with an amide in the E177Q variant completely inactivates XynII because the glutamine side chain cannot donate a proton to the xylosidic $\mathrm{O}$ atom to initiate the hydrolysis reaction. Co-crystallization of the E177Q variant with the xylohexaose oligosaccharide trapped the intact substrate molecule in the enzyme active site to give a pseudoMichaelis complex E177Q-X6 (Supplementary Table S3). All six xylose subunits of the substrate are clearly visible in the electron-density maps and are bound at subsites $-3,-2,-1,+1,+2$ and +3 (Fig. 4a). Superposition of E177Q$\mathrm{X} 6$ on the ligand-free E177Q structure that we determined at $1.5 \AA$ resolution showed minimal changes in the overall enzyme geometry, with an r.m.s.d. on $\mathrm{C}^{\alpha}$ atoms of $0.5 \AA$. The largest conformational change induced by substrate binding is in the 'thumb' region of the enzyme, as depicted in Supplementary Fig. S4. The thumb comprising residues 126-131 is drawn closer to the substrate in E177Q-X6 by about $2 \AA$ relative to its position in ligand-free E177Q and forms close interactions with the xylose subunits at subsites $-1,-2$ and -3 . The movement of the thumb is consistent with previous structural studies of XynII in complex with epoxyalkyl xylosides (Havukainen et al., 1996), but is in contrast to structures of $B$. subtilis and $A$. niger xylanases, which showed no movement of the thumb residues towards the ligands. The conformational variations of the thumb residues can be attributed to the differences in crystal packing. The thumb regions in the structures of the B. subtilis and A. niger xylanases (Vandermarliere et al., 2008) make strong hydrogen-bonding interactions with symmetry-related molecules, whereas in XynII the thumb 


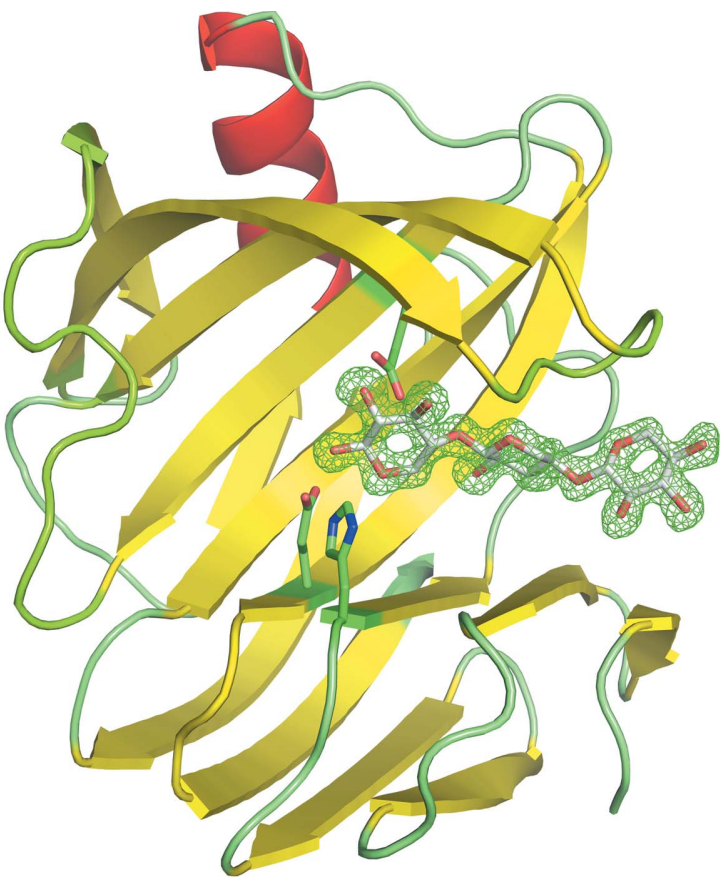

(a)

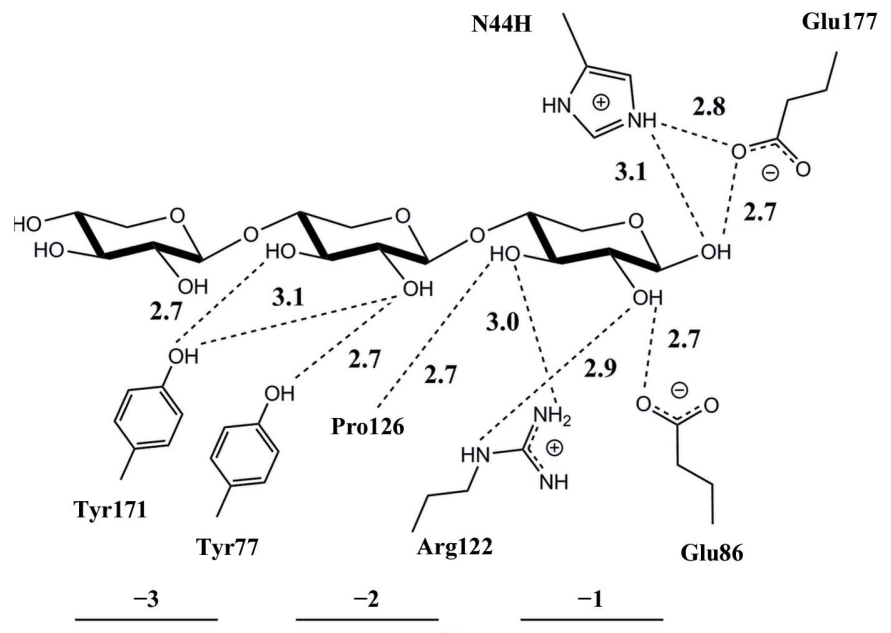

(b)
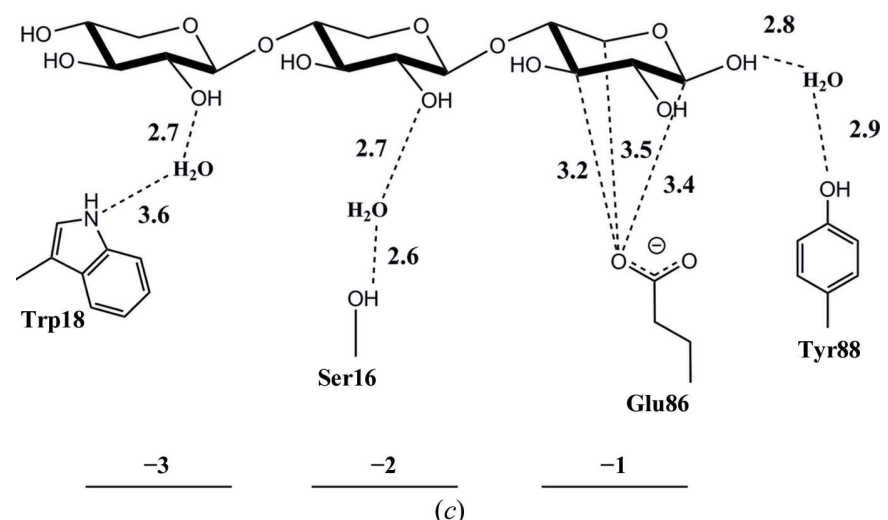

Figure 5

(a) OMIT difference $2 F_{\mathrm{o}}-F_{\mathrm{c}}$ electron density of xylotriose in $\mathrm{N} 44 \mathrm{H}-\mathrm{X} 3$ contoured at the $1.0 \sigma$ level. (b) Chemical diagram of conventional direct hydrogen-bond interactions between $\mathrm{X} 3$ and $\mathrm{N} 44 \mathrm{H}$ residues. (c) Watermediated interactions between $\mathrm{X} 3$ and the active-site residues and the unconventional $\mathrm{C}-\mathrm{H} \cdots \mathrm{O}$ hydrogen bonds between Glu86 and the -1 xylose subunit in $\mathrm{N} 44 \mathrm{H}-\mathrm{X} 3$. region is further than $4 \AA$ away from symmetry-related atoms and thus is able to alter its conformation upon substrate binding.

X6 makes numerous interactions with the active-site residues in E177Q-X6 (Figs. $4 b$ and $4 c$ ), including conventional and unconventional $(\mathrm{C}-\mathrm{H} \cdots \mathrm{O})$ hydrogen bonds, watermediated interactions, weaker $\mathrm{C}-\mathrm{H} \cdots \pi$ contacts and other hydrophobic contacts. The scissile xylosidic $\mathrm{O}$ atom connecting the xylose subunits in positions -1 and +1 is hydrogen-bonded to the $\mathrm{Gln} 177 \mathrm{NH}_{2}$ group with an $\mathrm{N} \cdots \mathrm{O}$ distance of $2.9 \AA$, mimicking the interaction in the physiological Michaelis complex. The nucleophile Glu86 makes a hydrogen bond to the $\mathrm{OH}$ group at $\mathrm{C} 2$ and several $\mathrm{C}-\mathrm{H} \cdots \mathrm{O}$ contacts with the $\mathrm{C}$ atoms of xylose in position -1 . In particular, there is a $\mathrm{C}-\mathrm{H} \cdots \mathrm{O}$ interaction of $3.3 \AA$ between the carboxylate group of Glu86 and the anomeric $\mathrm{C} 1$ of the xylose at site -1 ; thus, Glu86 is poised to directly attack $\mathrm{C} 1$ to produce the covalent intermediate. Hydrogen bonds between the $\mathrm{OH}$ substituent at $\mathrm{C} 2$ and the side chain of $\operatorname{Arg} 122$ and between the $\mathrm{OH}$ at $\mathrm{C} 3$ and the main-chain carbonyl of Pro126 further enhance the binding of this xylose subunit. The binding of xylose subunits in positions -2 and +1 is stabilized by several direct hydrogen bonds to four tyrosine residues (Tyr73, Tyr77, Tyr88 and Tyr171) and Gln136. The remaining xylose subunits (at subsites $-3,+2$ and +3 ) form fewer contacts with E177Q residues and therefore may be less strongly bound in the active site than those at subsites $-1,-2$ and +1 . Trp18 makes $\mathrm{C}-\mathrm{H} \cdots \pi$ contacts with the xylose rings at subsites -2 and -1 , with carbon. . carbon separations as short as 3.6-3.8 $\AA$ from the $\mathrm{CH}_{2}$ group at $\mathrm{C} 5$. At the glycone side of the substrate Tyr96 and Tyr179 sandwich the xylose at subsite +2 , whereas $\operatorname{Tyr} 96$ also forms $\mathrm{C}-\mathrm{H} \cdots \pi$ interactions with the xylose at subsite +3 . Again, the shortest distances of 3.7-3.8 $\AA$ are to the $\mathrm{CH}_{2}$ groups of $\mathrm{C} 5$ for both glycone xyloses. The xylose subunits at subsites $-2,-1$ and +1 have the lowest average $B$ factors; these increase twofold for the subunits at subsites $-3,+2$ and +3 . This indicates a tighter binding of the xylose subunits to the inner subsites than to the peripheral subsites. However, the $B$ factors for the xylose subunits at subsites -3 and +3 are only $15-18 \AA^{2}$. In addition, our observation of strong electron density for these xylose rings indicates that their conformational freedom is sufficiently reduced by intermolecular interactions with the enzyme.

\subsection{Structure of the $\mathrm{N} 44 \mathrm{H}-\mathrm{X} 3$ glycone complex}

As described above, the $\mathrm{N} 44 \mathrm{H}$ variant was substantially less active than the WT. N44H was co-crystallized with the X6 oligomer to give the glycone product $\mathrm{X} 3$, which is trapped within the active site. The electron density for $\mathrm{X} 3$ in this $\mathrm{N} 44 \mathrm{H}-\mathrm{X} 3$ complex is very clear, as shown in Fig. 5(a), while there is no interpretable electron density for the aglycone product. The latter therefore probably dissociates from the active site into the bulk solvent. Similar to the E177Q-X6 pseudo-Michaelis complex structure, $\mathrm{N} 44 \mathrm{H}-\mathrm{X} 3$ superimposes well on the ligand-free N44H structure (Supplementary Table 


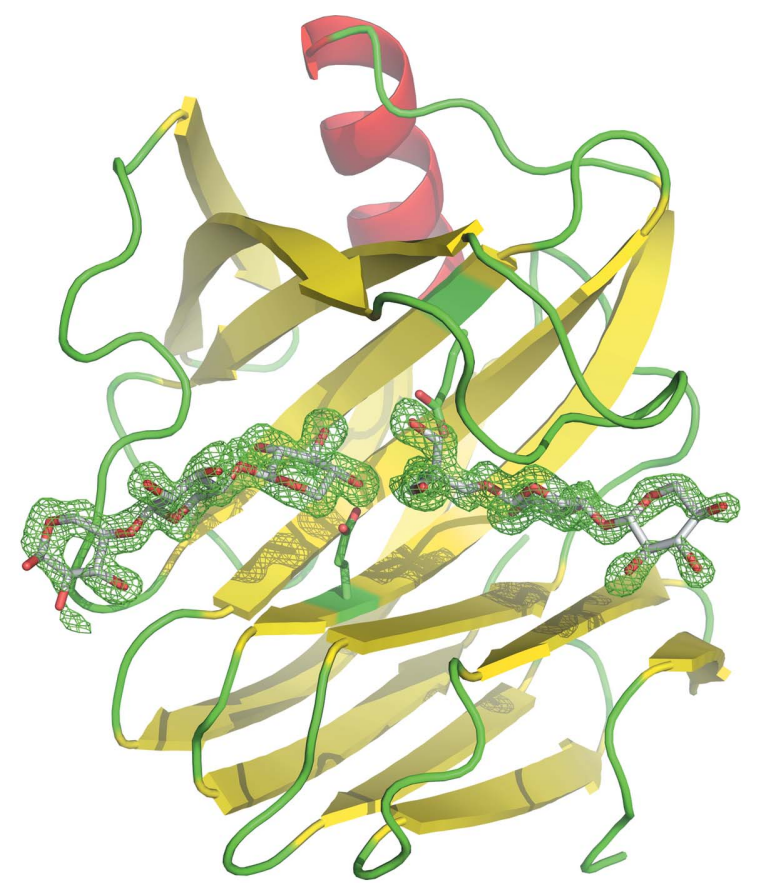

(a)

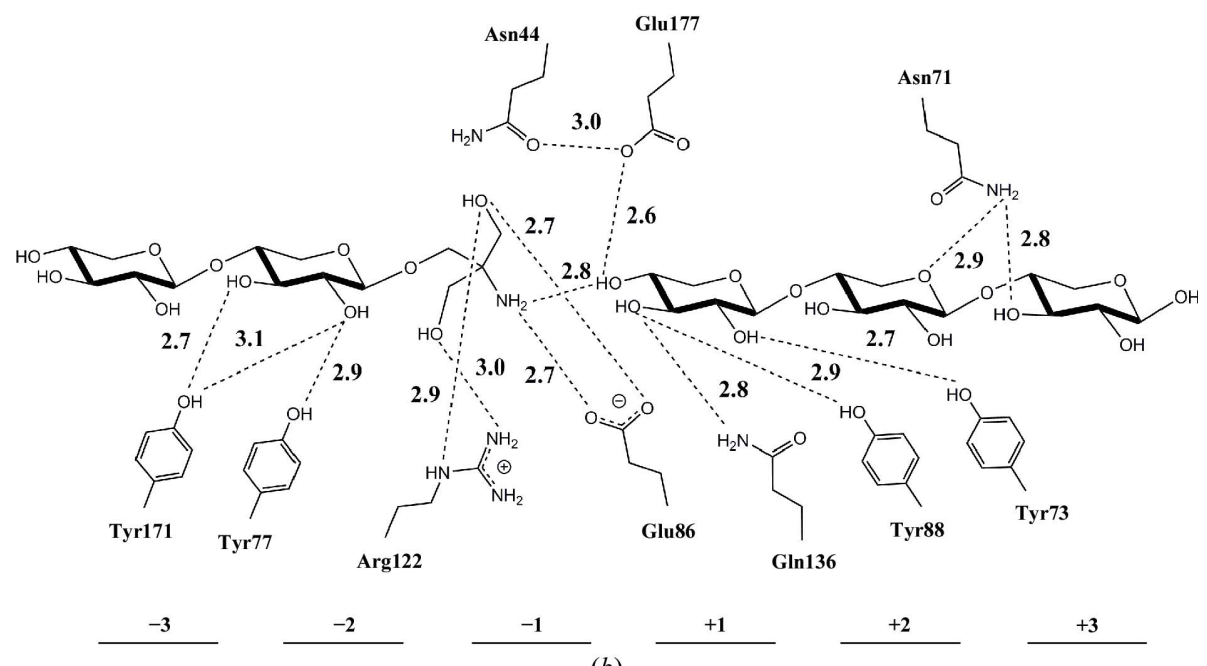

(b)

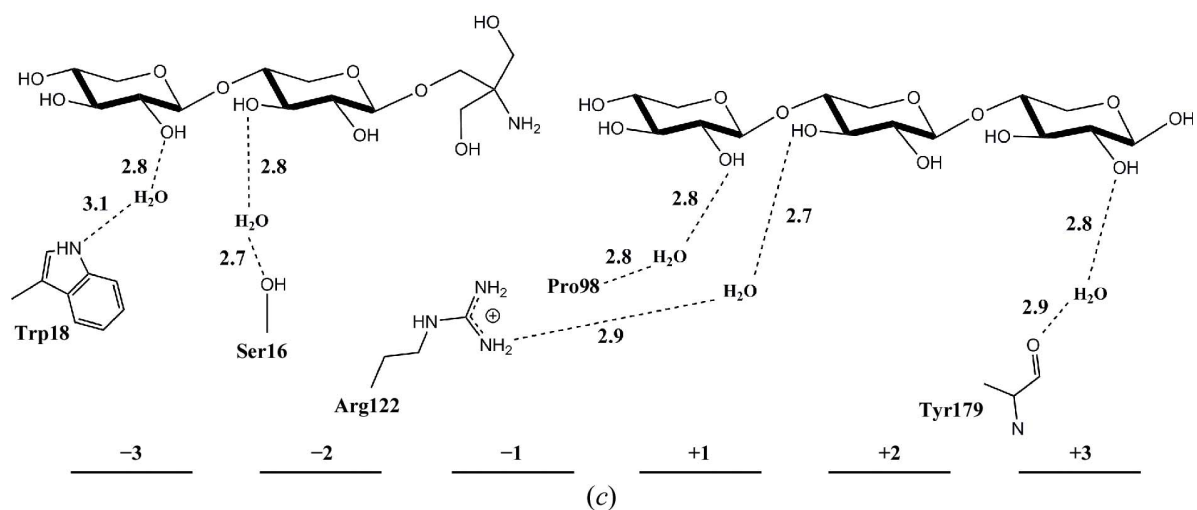

Figure 6

(a) OMIT difference $2 F_{\mathrm{o}}-F_{\mathrm{c}}$ electron density of Tris-xylobiose and xylotriose in native XynIITrisX2-X3 contoured at the $1.0 \sigma$ level. (b) Chemical diagram of conventional direct hydrogen-bond interactions between TrisX2, X3 and the residues of the enzyme. (c) Water-mediated interactions between ligands and the active-site residues in XynII-TrisX2-X3.
S3), with an r.m.s.d. on $\mathrm{C}^{\alpha}$ atoms of $0.5 \AA$, indicating that no large conformational motion is associated with the trapping of the product. The only significant conformational change is in the thumb region (Supplementary Fig. S5), where the atoms shift closer to the product relative to their positions in the ligand-free $\mathrm{N} 44 \mathrm{H}$ structure.

X3 occupies the sugar-binding subsites $-1,-2$ and -3 . The atoms of the product reside in almost exactly the same positions as the analogous atoms of the X6 substrate. Consequently, the coincident atoms from X3 and X6 make similar interactions with the active-site residues (Fig. $5 b$ and $5 c$ ). The only significant difference is a hydrogen bond of $3.1 \AA$ formed between the $\mathrm{OH}$ of $\mathrm{C} 1$ of the product and the imidazole ring of His44. An analogous hydrogen bond between the scissile $\mathrm{O}$ atom and Asn44 is absent in E177Q-X6. Other weaker interactions are similar to those found in the pseudo-Michaelis complex.

\subsection{Structure of the XynII-TrisX2-X3 complex}

To obtain the XynII-TrisX2-X3 complex, the native enzyme was cocrystallized with xylopentaose oligosaccharide at a basic $\mathrm{pH}$ of 8.5 in Tris buffer. The expectation was that either a xylobiose or xylotriose glycone product would be trapped, as observed in the $\mathrm{N} 44 \mathrm{H}-\mathrm{X} 3$ complex. Instead, surprisingly, we observed the glycone product of a transglycosylation reaction, TrisX2, in subsites $-1,-2$ and -3 and the aglycone xylotriose in subsites $+1,+2$ and +3 . Both products were clearly visible in the electron-density maps calculated from the X-ray data at $1.65 \AA$ resolution, as shown in Fig. 6(a). TrisX2 must have formed when a Tris buffer molecule attacked the $\mathrm{C} 1$ atom of the covalent intermediate with one of its hydroxyl groups. The Tris substituent pushed the glycone xylobiose along the length of the XynII active site to subsites -2 and -3 . As a result, the Tris group is positioned at subsite -1 . Similar to the other complexes described above, the thumb residues of XynII-TrisX2-X3 move in closer to the products (Supplementary Fig. S6) rela- 
tive to their positions in the ligand-free XynII structure (PDB entry 2dfb; Törrönen \& Rouvinen, 1995).

When XynII-TrisX2-X3 is superimposed on the pseudoMichaelis complex E177Q-X6, the sugar subunits occupy almost exactly the same positions. Consequently, their interactions with XynII are very similar in both structures (Figs. $4 b$, $6 b$ and $6 c$ and Supplementary Fig. S7). The two free $\mathrm{OH}$ groups of Tris also make hydrogen bonds to the side chains of Arg122 and Glu86 in much the same fashion as the xylose at subsite -1 of the substrate oligosaccharide. The $\mathrm{OH}$ at $\mathrm{C} 4$ of the aglycone xylotriose stays hydrogen-bonded to Glu177 after the bond to the glycone has been cleaved. In XynIITris $\mathrm{X} 2-\mathrm{X} 3$ this $\mathrm{OH}$ substituent also gains a hydrogen bond to the amino group of Tris.

\section{Discussion}

In the current study, we modulated the activity of XynII by amino-acid replacements and $\mathrm{pH}$ adjustment to obtain several complexes along the hydrolysis and transglycosylation pathways of the enzyme. By completely abolishing activity using the E177Q substitution, which prevents substrate protonation, we were able (for the first time for a family 11 xylanase) to obtain a pseudo-Michaelis complex E177Q-X6. In the atomic resolution structure of E177Q-X6 all six xylose units of the substrate xylohexaose were clearly visible in the electrondensity maps. A previous structure of a ternary complex of a family 11 xylanase bound to xylobiose and a xylotriose analog suggested that the protein contained six carbohydrate-binding subsites; however, disorder in the active site prevented detailed analysis (Vardakou et al., 2008). Another crystallographic study demonstrated that inactive variants of the $B$. subtilis and A. niger xylanases could bind oligosaccharide substrates at subsites -3 through +1 (Vandermarliere et al., 2008). Previous experiments demonstrated that family 11 xylanases preferentially bind longer chain thioxylooligosaccharides (Jänis et al., 2007) and the specificity constant of the enzyme for xylohexaose hydrolysis was almost 12-fold higher than that for xylopentaose hydrolysis (Vardakou et al., 2008). These results indicate that these xylanases have six sugar-binding subsites. The current study provided unambiguous structural evidence that the active sites of XynII, and perhaps all family 11 xylanases, have six possible sugar-binding subsites from -3 through +3 . The evidence is corroborated by the presence of numerous intermolecular interactions between all six xylose subunits and the residues of the enzyme and the fact that the sugars have low atomic displacement parameters.

In the E177Q-X6 structure, the side-chain amide of Gln177 forms a hydrogen bond of $2.9 \AA$ to the xylosidic exocyclic $\mathrm{O}$ atom connecting xylose subunits -1 and +1 (Fig. $4 b$ ), mimicking a putative interaction of the catalytic glutamic acid in the actual Michaelis complex. There is substantial evidence that this glutamate residue has an elevated $\mathrm{p} K_{\mathrm{a}}$ value of $\sim 7$ and is able to donate a proton to the $\mathrm{O} 1$ atom of the leaving group (Miao et al., 1994; Davoodi et al., 1995; McIntosh et al., 1996), although protonation of the Glu177 carboxylate has not directly been observed. The same $\mathrm{Gln} 177$ amide is also hydrogen-bonded to the side-chain $\mathrm{O}$ atom of Asn44, with an $\mathrm{N}$...O distance of $2.7 \AA$. This interaction is absent in the ligand-free E177Q structure, where the $\mathrm{N} \cdots \mathrm{O}$ separation is $4 \AA$. Therefore, substrate binding induces a shift of Asn44 closer to residue 177 and the substrate. The three Asn44 substitutions substantially impaired the xylan hydrolytic activity by decreasing the turnover and increasing the cooperativity (N44D), by destabilizing the Michaelis complex $(\mathrm{N} 44 \mathrm{~V})$ or by introducing new nonproductive substrate interactions $(\mathrm{N} 44 \mathrm{H})$. Although the $\mathrm{N} 44 \mathrm{D}$ variant demonstrated the predicted enhanced relative activity at acidic $\mathrm{pH}$, it differed from the corresponding N35D variant of B. circulans, which demonstrated greater hydrolytic activity than WT using $\mathrm{PNPX}_{2}$ substrate (Joshi et al., 2000). The three Asn44 variants retained significant $\mathrm{PNPX}_{2}$ hydrolytic activity, indicating that the rate of $p$-nitrophenol leaving-group release is less dependent on the protonation state of Glu177 than natural hydroxyl leaving groups without electron delocalization. If the interaction of Glu177 with Asn44 forms in the Michaelis complex of the native enzyme, it might provide the driving force to initiate hydrolysis by activating Glu177 to donate a proton to the substrate. Other substitutions of nearby aminoacid residues that were predicted to increase the active-site hydrophobicity (V46L, A175S and A175V) produced enzymes with higher relative xylanase activity at alkaline $\mathrm{pH}$, probably owing to an increase in the apparent $\mathrm{p} K_{\mathrm{a}}$ of the Glu177 carboxylate.

The mechanism of positive kinetic cooperativity during xylan hydrolysis observed for the WT and most variant XynII proteins requires further study. Allosteric binding sites cause cooperative behavior in some proteins. The B. circulans xylanase has been reported to bind xylooligosaccharides at a second site and was found to function cooperatively in binding long oligosaccharides and xylan (Ludwiczek et al., 2007). Complexes of $B$. subtilis and $A$. niger variant xylanases were crystallized with xylooligosaccharides bound both at the active site and at disparate surface binding sites (Vandermarliere $e t$ al. 2008). A model for the cooperative binding of xylan to the B. circulans xylanase during the catalytic cycle has also been proposed (Ludwiczek et al., 2007). Although sigmoidal substrate-saturation curves are often attributed to second-site binding or to cooperativity between protein subunits, some monomeric enzymes also demonstrate nonhyperbolic kinetics (Porter \& Miller, 2012). The monomeric glucokinase enzyme demonstrates this cooperativity in binding glucose, an effect that is attributed to a slow transition in the conformation of two domains (Kamata et al., 2004). According to this model, high levels of glucose keep glucokinase in the active conformation, while it can slowly adopt an inactive conformation in the absence of substrate. Xylanase also undergoes a conformational change in the thumb region owing to substrate binding, although the rates of this movement are not known. The kinetic cooperativity of XynII could thus be explained by the multi-site binding of xylan, or it could also be related to the rate of conformational change of the enzyme. 
The N44H substitution substantially diminished the xylanase activity, which allowed trapping of the glycone xylotriose product in the $\mathrm{N} 44 \mathrm{H}-\mathrm{X} 3$ binary complex for crystallography. The xylotriose occupied binding sites -1 through -3 , whereas there was no indication of the presence of the reducing aglycone product at sites +1 through +3 . Co-crystallization of the native XynII with xylopentaose in Tris buffer at an alkaline $\mathrm{pH}$ (8.5) that inhibits the hydrolytic activity of the enzyme promoted a parallel reaction in which the hydroxyl group of the buffer molecule attacked the $\mathrm{C} 1$ atom of the glycone product after cleavage of the xylosidic bond to generate the transglycosylation product TrisX2. Moreover, the aglycone xylotriose product was observed bound together with the transglycosylation product in the active site of the XynIITrisX2-X3 ternary complex, perhaps stabilized by a hydrogen bond to the Tris amino group.

The E177Q-X6 structure provides a snapshot of the active site and the substrate before the general acid protonates the xylosidic $\mathrm{O}$ atom to initiate the hydrolysis reaction. The active site of the enzyme appears to be asymmetric so that the X6 oligosaccharide binds only in one direction, with the glycone end positioned near the peripheral residues Trp18 and Tyr171, and the aglycone end near Tyr179. We explored the possibility that X6 may have bound in the reverse direction because the sugar rings superimpose exactly on each other when the substrate molecule is rotated by $180^{\circ}$ around the direction perpendicular to the long axis of the oligosaccharide molecule. Only the $\mathrm{C} 5$ and $\mathrm{O} 5$ positions are reversed by this rotation. Owing to the atomic resolution of the E177Q-X6 structure this rotation leads to the appearance of extra positive and negative $F_{\mathrm{o}}-F_{\mathrm{c}}$ difference electron density above the $3 \sigma$ level at the new C5 and O5 positions, respectively (Supplementary Fig. S8). This observation indicates that an atom with fewer electrons (C5) has been placed at a position in which a more electron-rich atom (O5) should be located. Conversely, negative difference electron density appeared when O5 was incorrectly placed at the position of C5. This rotation would also disrupt the specific hydrogen-bonding interactions made by the endocyclic xylose $\mathrm{O}$ atoms. For example, O5 of the xylose unit at subsite +2 makes a direct hydrogen bond of $3.0 \AA$ to the side-chain amide of Asn71 (Fig. $4 b$ ). O5 of the xylose unit at subsite -3 makes a hydrogen bond of $2.7 \AA$ to a well defined water molecule. Also, the $\mathrm{O} 5$ atoms of the xylose units at subsites -1 and +1 form weak hydrogen-bonding contacts of $3.3 \AA$ to the Gln 177 side chain and to a water molecule, respectively. If the substrate molecule were to flip its orientation in the active site then these hydrogen bonds would be replaced either by weaker $\mathrm{C}-\mathrm{H}$. . O contacts or possibly by repulsive $\mathrm{C}-\mathrm{H} \cdots \mathrm{H}-\mathrm{N}$ interactions. Moreover, superposition of the ligands in E177Q-X6, N44H-X3 and XynIITris $\mathrm{X} 2-\mathrm{X} 3$ suggests that all of them are bound in the same direction along the enzyme active site. Owing to all of these factors, we rejected the model in which the xylohexaose orientation was reversed in the E177Q-X6 binary complex.

Glycoside hydrolases stabilize oxocarbenium-ion-like transition states that promote leaving-group departure at the anomeric $\mathrm{C} 1$ position by binding sugars at subsite -1 with ring conformations that are distorted from the relaxed ${ }^{4} C_{1}$ conformation (Davies et al., 2003, 2012; Biarnés et al., 2010). In crystal structures of the enzymes from various families the sugar at subsite -1 was observed in a number of different conformations, which were found to be specific to the enzyme family and species (i.e. sequence). Table 2 shows the CremerPople ring-puckering parameters (Jeffrey \& Yates, 1979) for all xylose rings in the three ligand-bound structures reported here. The majority of the rings have the common ${ }^{4} C_{1}$ chair conformation. The xylose ring occupying subsite -1 , however, adopts a conformation that is distorted from this global energy minimum geometry in both the E177Q-X6 substrate and the $\mathrm{N} 44 \mathrm{H}-\mathrm{X} 3$ glycone product complexes. The $\Theta$ angle for this sugar subunit is close to $30^{\circ}$ in the substrate and product molecules, indicating a significant departure from ${ }^{4} C_{1}$ towards the ${ }^{\mathrm{O}} E /{ }^{\mathrm{O}} C_{3}$ distorted envelope conformation. The endocyclic oxygen $\mathrm{O} 5$ is substantially out of the plane made by the $\mathrm{C} 1$, $\mathrm{C} 2, \mathrm{C} 4$ and $\mathrm{C} 5$ atoms, with the $\mathrm{C} 3$ atom being very slightly out of this plane. The aberrant dihedral angles $(\varphi$ and $\psi)$ formed between the -1 and +1 xyloses indicate that the linkage between them is twisted to facilitate hydrolysis. In the pseudoMichaelis complex of $B$. subtilis family 11 xylanase, the xylose residue at subsite -1 was distorted towards the ${ }^{2} S_{\mathrm{O}}$ skew conformation (Vandermarliere et al., 2008). In all covalent intermediate structures of several family 11 xylanases reported to date this xylose residue adopted the ${ }^{2,5} B$ boat conformation (Sidhu et al., 1999; Sabini et al., 1999, 2001). Our observation of the ${ }^{\mathrm{O}} E /{ }^{\mathrm{O}} C_{3}$ xylose conformation is in accord with the previously suggested conformational itinerary for family 11 xylanases, in which the geometry of the substrate should be distorted towards the $E_{3}$ conformation in the transition state before the xylosidic bond is cleaved (Nerinckx et al., 2006). Thus, to generate the $E_{3}$ conformation O5 is brought into the plane of $\mathrm{C} 1, \mathrm{C} 2, \mathrm{C} 4$ and $\mathrm{C} 5$, with no other geometry changes in the pyranose ring. The difference in the conformation of xylose at subsite -1 in our E177Q-X6 structure and that of B. subtilis xylanase could be owing to the inactivating E172A substitution in the latter protein. The catalytic general acid/base glutamate was substituted by alanine in $B$. subtilis xylanase, effectively removing the hydrogen-bonding capability and steric effects of this residue. Our E177Q mutation preserved the ability of the side chain to form hydrogen bonds and maintained its steric size. Hence, our E177Q-X6 structure and the geometry of the xylose rings may demonstrate a more relevant substrate-bound intermediate in the reaction trajectory of the enzyme. In a recent QM/MM study of the entire hydrolysis reaction catalyzed by the xylanase Cex from Cellulomonas fimi, the xylose subunit at position -1 was found to adopt ${ }^{\mathrm{O}} S_{2}$ conformations in the transition states for both the glycosylation and the deglycosylation steps, whereas it had the $B_{2,5}$ conformation in the covalent intermediate complex (Liu et al., 2012). The ${ }^{\mathrm{O}} S_{2}$ conformation would be readily accessible from the observed ${ }^{\mathrm{O}} E /{ }^{\mathrm{O}} C_{3}$ conformation in $\mathrm{E} 177 \mathrm{Q}-\mathrm{X} 6$, whereas the $B_{2,5}$ conformation is adjacent to ${ }^{\mathrm{O}} S_{2}$ on the Stoddart diagram. Therefore, it is possible for the substrate bound to XynII to undergo similar conformational changes as found in xylanase 
Cex; that is, ${ }^{4} C_{1} \rightarrow{ }^{\mathrm{O}} E \rightarrow\left({ }^{\mathrm{O}} S_{2}\right)^{\ddagger} \rightarrow B_{2,5} \rightarrow\left({ }^{\mathrm{O}} S_{2}\right)^{\ddagger} \rightarrow{ }^{\mathrm{O}} E \rightarrow{ }^{4} C_{1}$. These proposed conformational iteneraries of the xylose ring at subsite -1 are depicted in Fig. 7 along with the possible reaction mechanism catalyzed by XynII.

The generally accepted catalytic cycle of polysaccharide hydrolysis assumes that the aglycone product leaves the active site before a water molecule attacks the glycone intermediate, because no aglycone product has been observed in covalent intermediate or product complexes, including $\mathrm{N} 44 \mathrm{H}-\mathrm{X} 3$ as studied here. In contrast, we were able to trap the aglycone product in the XynII-TrisX2-X3 structure at alkaline $\mathrm{pH}$, which also contained a product of the transglycosylation reaction between the glycone and a Tris buffer molecule. The Tris molecule apparently attacked the $\mathrm{C} 1$ of the covalent intermediate, pushed the xylobiose portion to subsites -2 and -3 and occupied subsite -1 . The observation of both products in the XynII active site may indicate that attack on the intermediate glycosyl-enzyme state can occur before the aglycone product leaves the active site. It is also possible that the aglycone $\mathrm{X} 3$ first dissociates from the active site, allowing the transglycosylation reaction to take place and then rebinds, driven by the formation of hydrogen bonds between its scissile xylosidic $\mathrm{O}$ atom that became $\mathrm{O} 4$ and the amino group of Tris and the carboxylate group of Glu177 (Gäb et al., 2010; Fig. 6b).
Although transglycosylation is a common reaction catalyzed by glycoside hydrolases, this is the first observation of the product bound to the active site of a xylanase.

In summary, we have generated XynII variants by substituting positions adjacent to the catalytic residues and were able to modulate the $\mathrm{pH}$ dependence of the activity of the enzyme. In particular, the V46L variant demonstrated optimal activity towards beechwood xylan at basic $\mathrm{pH}$, whereas the activity of the A175S variant was independent of $\mathrm{pH}$ between $\mathrm{pH} 4$ and $\mathrm{pH}$ 7. The E177Q-X6 structure is the first family 11 xylanase pseudo-Michaelis complex that unequivocally establishes the presence of six sugar-binding subsites. The distortion of the ring towards the ${ }^{\mathrm{O}} E /{ }^{\mathrm{O}} C_{3}$ conformation of the xylose subunit at the glycone subsite -1 observed in the E177Q-X6 and N44H-X3 structures differs from the conformations found in the structures of other family 11 and family 10 enzymes, expanding the conformational itinerary of this xylose ring. Our observation of the glycone tranglycosylation product trapped together with the aglycone product in the active site of XynII may indicate that attack on the glycone $\mathrm{C} 1$ by an incoming water or alcohol molecule may be possible before the leaving group (aglycone) of the first reaction stage has had time to dissociate from the enzyme active site.
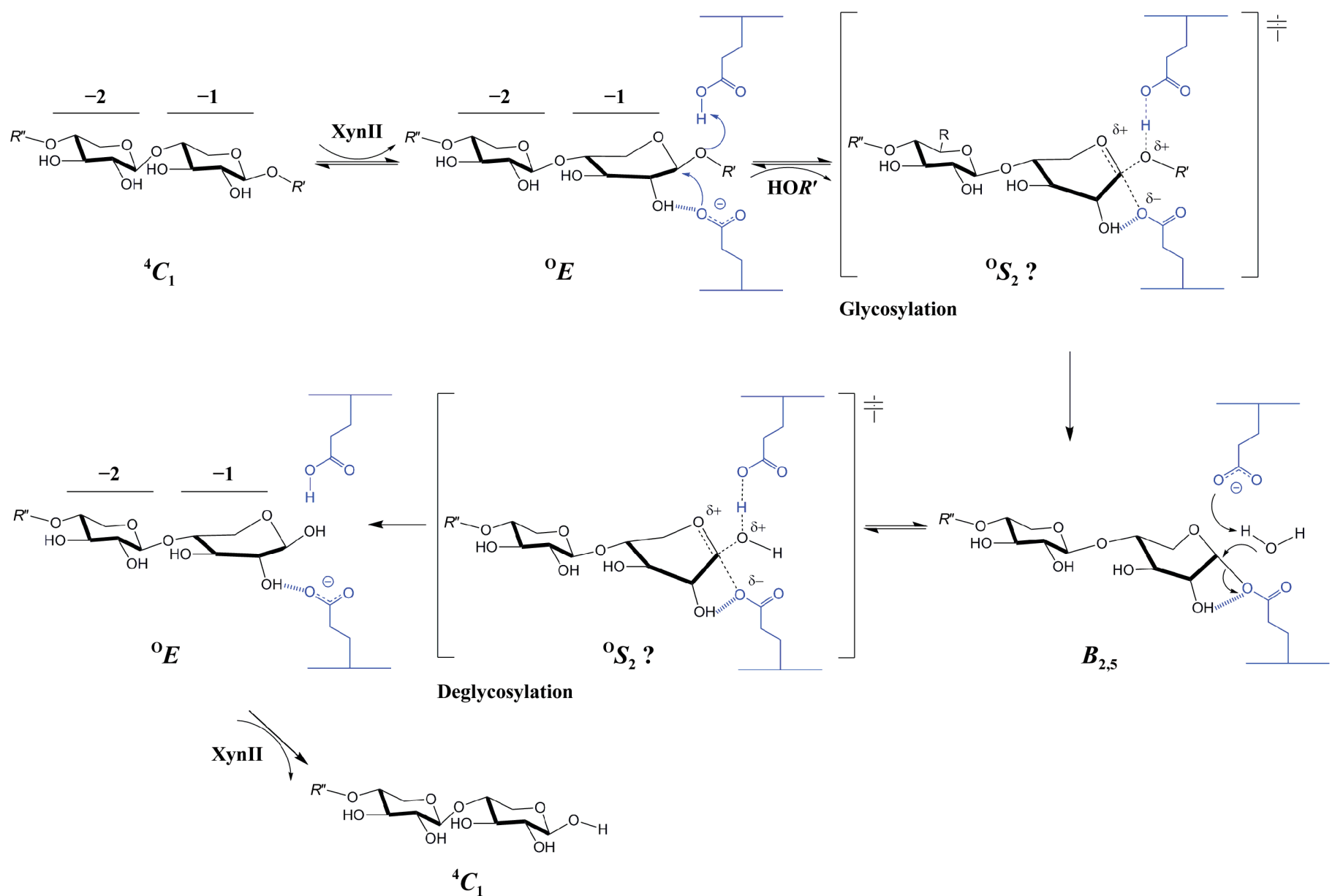

Figure 7

The proposed chemical mechanism of xylan hydrolysis catalyzed by XynII. The possible conformational itinerary of the xylose ring at subsite -1 is shown based on the E177Q-X6 structure, the covalent intermediate structure reported previously and recent QM/MM calculations. 
This research was supported by the Laboratory Directed Research and Development Program (LDRD) at Oak Ridge National Laboratory, which is managed by UT-Battelle LLC for the US Department of Energy's Office of Science under contract No. DE-AC05-00OR22725. The Office of Biological and Environmental Research supported research at the Oak Ridge National Laboratory Center for Structural Molecular Biology (CSMB) using facilities supported by the Scientific User Facilities Division, Office of Basic Energy Sciences, United States Department of Energy. LC, PL and AK were partly supported by the Office of Basic Energy Sciences, United States Department of Energy. We thank the members of the 19ID beamline at the Advanced Photon Source at Argonne National Laboratory for assistance with data collection. Argonne is operated by UChicago Argonne LLC for the US Department of Energy's Office of Science under contract DE-AC02-06CH11357. Notice: This manuscript has been authored by UT-Battelle LLC under Contract No. DEAC05-00OR22725 with the US Department of Energy.

\section{References}

Adams, P. D. et al. (2010). Acta Cryst. D66, 213-221.

Afonine, P. V., Grosse-Kunstleve, R. W., Echols, N., Headd, J. J., Moriarty, N. W., Mustyakimov, M., Terwilliger, T. C., Urzhumtsev, A., Zwart, P. H. \& Adams, P. D. (2012). Acta Cryst. D68, 352-367.

Bailey, M. J., Biely, P. \& Poutanen, K. (1992). J. Biotechnol. 23, 257-270.

Biarnés, X., Ardèvol, A., Planas, A. \& Rovira, C. (2010). Biocatal. Biotransform. 28, 33-40.

Chen, V. B., Arendall, W. B., Headd, J. J., Keedy, D. A., Immormino, R. M., Kapral, G. J., Murray, L. W., Richardson, J. S. \& Richardson, D. C. (2010). Acta Cryst. D66, 12-21.

Collins, T., Gerday, C. \& Feller, G. (2005). FEMS Microbiol. Rev. 29, $3-23$.

Davies, G. J., Ducros, V. M., Varrot, A. \& Zechel, D. L. (2003). Biochem. Soc. Trans. 31, 523-527.

Davies, G. \& Henrissat, B. (1995). Structure, 3, 853-859.

Davies, G. J., Planas, A. \& Rovira, C. (2012). Acc. Chem. Res. 45, 308-316.

Davies, G. J., Wilson, K. S. \& Henrissat, B. (1997). Biochem. J. 321, 557-559.

Davoodi, J., Wakarchuk, W. W., Campbell, R. L., Carey, P. R. \& Surewicz, W. K. (1995). Eur. J. Biochem. 232, 839-843.

De Vos, D., Collins, T., Nerinckx, W., Savvides, S. N., Claeyssens, M., Gerday, C., Feller, G. \& Van Beeumen, J. (2006). Biochemistry, 45, 4797-4807.

Dodd, D. \& Cann, I. K. O. (2009). Glob. Change Biol. Bioenerg. 1, 2-17.

Drevland, R. M., Waheed, A. \& Graham, D. E. (2007). J. Bacteriol. 189, 4391-4400.

Emsley, P., Lohkamp, B., Scott, W. G. \& Cowtan, K. (2010). Acta Cryst. D66, 486-501.

Fushinobu, S., Ito, K., Konno, M., Wakagi, T. \& Matsuzawa, H. (1998). Protein Eng. 11, 1121-1128.

Gäb, J., John, H., Melzer, M. \& Blum, M. (2010). J. Chromatogr. B, 878, 1382-1390.

Garlock, R. J., Chundawat, S. P., Balan, V. \& Dale, B. E. (2009). Biotechnol. Biofuels, 2, 29.

Gibbs, M., Reeves, R., Hardiman, E., Choudhary, P., Daniel, R. \& Bergquist, P. (2010). New Biotechnol. 27, 795-802.

Gupta, R., Kim, T. H. \& Lee, Y. Y. (2008). Appl. Biochem. Biotechnol. 148, 59-70.
Havukainen, R., Törrönen, A., Laitinen, T. \& Rouvinen, J. (1996). Biochemistry, 35, 9617-9624.

Jänis, J., Pulkkinen, P., Rouvinen, J. \& Vainiotalo, P. (2007). Anal. Biochem. 365, 165-173.

Jeffrey, G. A. \& Yates, J. H. (1979). Carbohydr. Res. 74, 319-322.

Joshi, M. D., Sidhu, G., Pot, I., Brayer, G. D., Withers, S. G. \& McIntosh, L. P. (2000). J. Mol. Biol. 299, 255-279.

Kamata, K., Mitsuya, M., Nishimura, T., Eiki, J. \& Nagata, Y. (2004). Structure, 12, 429-438.

Ko, E. P., Akatsuka, H., Moriyama, H., Shinmyo, A., Hata, Y., Katsube, Y., Urabe, I. \& Okada, H. (1992). Biochem. J. 288, 117-121.

Krissinel, E. \& Henrick, K. (2004). Acta Cryst. D60, 2256-2268.

Liu, J., Zhang, C. \& Xu, D. (2012). J. Mol. Graph. Model. 37, 67-76.

Ludwiczek, M. L., D’Angelo, I., Yalloway, G. N., Brockerman, J. A., Okon, M., Nielsen, J. E., Strynadka, N. C., Withers, S. G. \& McIntosh, L. P. (2013). Biochemistry, 52, 3138-3156.

Ludwiczek, M. L., Heller, M., Kantner, T. \& McIntosh, L. P. (2007). J. Mol. Biol. 373, 337-354.

McCarter, J. D. \& Withers, S. G. (1994). Curr. Opin. Struct. Biol. 4, 885-892.

McCoy, A. J., Grosse-Kunstleve, R. W., Adams, P. D., Winn, M. D., Storoni, L. C. \& Read, R. J. (2007). J. Appl. Cryst. 40, 658-674.

McIntosh, L. P., Hand, G., Johnson, P. E., Joshi, M. D., Körner, M., Plesniak, L. A., Ziser, L., Wakarchuk, W. W. \& Withers, S. G. (1996). Biochemistry, 35, 9958-9966.

Miao, S., Ziser, L., Aebersold, R. \& Withers, S. G. (1994). Biochemistry, 33, 7027-7032.

Nerinckx, W., Desmet, T. \& Claeyssens, M. (2006). ARKIVOC, 2006, 90-116.

Notenboom, V., Birsan, C., Nitz, M., Rose, D. R., Warren, R. A. J. \& Withers, S. G. (1998). Nature Struct. Mol. Biol. 5, 812-818.

Notenboom, V., Birsan, C., Warren, R. A. J., Withers, S. G. \& Rose, D. R. (1998). Biochemistry, 37, 4751-4758.

Otwinowski, Z. \& Minor, W. (1997). Methods Enzymol. 276, 307-326.

Porter, C. M. \& Miller, B. G. (2012). Bioorg. Chem. 43, 44-50.

Rantwijk, F. van, Woudenberg-van Oosterom, M. \& Sheldon, R. (1999). J. Mol. Catal. B Enzym. 6, 511-532.

Sabini, E., Sulzenbacher, G., Dauter, M., Dauter, Z., Jørgensen, P. L., Schülein, M., Dupont, C., Davies, G. J. \& Wilson, K. S. (1999). Chem. Biol. 6, 483-492.

Sabini, E., Wilson, K. S., Danielsen, S., Schülein, M. \& Davies, G. J. (2001). Acta Cryst. D57, 1344-1347.

Saff, E. B. \& Kuijlaars, A. B. J. (1997). Math. Intell. 19, 5-11.

Scheller, H. V. \& Ulvskov, P. (2010). Annu. Rev. Plant Biol. 61, 263-289.

Sidhu, G., Withers, S. G., Nguyen, N. T., McIntosh, L. P., Ziser, L. \& Brayer, G. D. (1999). Biochemistry, 38, 5346-5354.

Strynadka, N. C. \& James, M. N. G. (1996). EXS, 75, 185-222.

Suzuki, R., Fujimoto, Z., Ito, S., Kawahara, S., Kaneko, S., Taira, K., Hasegawa, T. \& Kuno, A. (2009). J. Biochem. 146, 61-70.

Taguchi, H., Hamasaki, T., Akamatsu, T. \& Okada, H. (1996). Biosci. Biotechnol. Biochem. 60, 983-985.

Törnkvist, M., Larsson, G. \& Enfors, S.-O. (1996). Bioprocess Biosyst. Eng. 15, 231-237.

Törrönen, A., Harkki, A. \& Rouvinen, J. (1994). EMBO J. 13, $2493-$ 2501.

Törrönen, A., Mach, R. L., Messner, R., Gonzalez, R., Kalkkinen, N., Harkki, A. \& Kubicek, C. P. (1992). Nature Biotechnol. 10, 14611465.

Törrönen, A. \& Rouvinen, J. (1995). Biochemistry, 34, 847-856.

Törrönen, A. \& Rouvinen, J. (1997). J. Biotechnol. 57, 137-149.

Vandermarliere, E., Bourgois, T. M., Rombouts, S., Van Campenhout, S., Volckaert, G., Strelkov, S. V., Delcour, J. A., Rabijns, A. \& Courtin, C. M. (2008). Biochem. J. 410, 71-79.

Vardakou, M., Dumon, C., Murray, J. W., Christakopoulos, P., Weiner, D. P., Juge, N., Lewis, R. J., Gilbert, H. J. \& Flint, J. E. (2008). J. Mol. Biol. 375, 1293-1305. 


\section{research papers}

Vasella, A., Davies, G. J. \& Böhm, M. (2002). Curr. Opin. Chem. Biol. 6, 619-629.

Wakarchuk, W. W., Campbell, R. L., Sung, W. L., Davoodi, J. \& Yaguchi, M. (1994). Protein Sci. 3, 467-475.

Wan, Q., Kovalevsky, A., Zhang, Q., Hamilton-Brehm, S., Upton, R., Weiss, K. L., Mustyakimov, M., Graham, D., Coates, L. \& Langan, P. (2013). Acta Cryst. F69, 320-323.
Watanabe, N., Akiba, T., Kanai, R. \& Harata, K. (2006). Acta Cryst. D62, 784-792.

White, A. \& Rose, D. R. (1997). Curr. Opin. Struct. Biol. 7, 645-651. Winn, M. D. et al. (2011). Acta Cryst. D67, 235-242.

Zechel, D. L. \& Withers, S. G. (2000). Acc. Chem. Res. 33, 11-18.

Zolotnitsky, G., Cogan, U., Adir, N., Solomon, V., Shoham, G. \& Shoham, Y. (2004). Proc. Natl Acad. Sci. USA, 101, 11275-11280. 